\title{
Análise dos recursos terapêuticos em pacientes idosos com osteoartrite do joelho:
}

\section{revisão de literatura}

Analysis of therapeutic resources in elderly patients with knee osteoarthritis: literature review

Análisis de recursos terapéuticos en pacientes ancianos con artrosis de rodilla: revisión de la

\section{literatura}

Recebido: 10/09/2021 | Revisado: 18/09/2021 | Aceito: 08/10/2021 | Publicado: 09/10/2021

\author{
Artur Victor de Oliveira Paixão da Cunha \\ ORCID: https://orcid.org/0000-0002-2958-2628 \\ Instituto de Pós-graduação e Graduação, Brasil \\ E-mail: avopc@ hotmail.com \\ Jeniffer Santos Mesquita \\ ORCID: https://orcid.org/0000-0003-1393-4841 \\ Faculdade Unyleya, Brasi \\ E-mail: jeniffersm3@yahoo.com.br
}

\begin{abstract}
Resumo
A osteoartrite (OA) de joelho é uma patologia de ordem degerenativa, que ocorre por causa de diversos fatores. No mundo, existem 50 milhões de pessoas nessa condição. A fisioterapia, possui diversas técnicas para tratar esses indivíduos, dentre as já conhecidas: eletrofototermoterapia e cinesioterapia. O estudo busca abordagens que possam ser aplicadas na prática clínica baseada em evidências. A pesquisa surge através da estratégia PICO: idosos, recursos terapêuticos, tratamento conservador, osteoartrite do joelho. Aplicando operador booleano AND para maximizar os resultados, foram encontrados duzentos e sessenta e oito artigos nas bases Google Scholar, Scielo e MEDLINE/PubMed. Os resultados foram inseridos em quadros separados para melhor didática e entendimento das pesquisas. Através do presente estudo concluí-se que a fisioterapia possui recursos muito mais amplos do que a eletrotermofototerapia e cinesioterapia. Além disso, todos os recursos achados podem ser aplicados dentro e fora do ambiente da clínica de fisioterapia com objetivos específicos quanto ao grau de OA.
\end{abstract}

Palavras-chave: Idoso; Osteoartrite de joelho; Fisioterapia.

\begin{abstract}
Knee osteoarthritis (OA) is a pathology of a degenerative order, which occurs because of several factors. In the world there are 50 million people in this condition, physiotherapy has several techniques to treat these individuals among those already known, electrophotothermotherapy and kinesiotherapy. The study seeks approaches that can be applied in evidence-based clinical practice. The research emerges through the PICO strategy: Elderly, therapeutic resources, conservative treatment, osteoartitis of the knee. Applying a Boolean AND operator to maximize results, with 268 articles found in Google Scholar, Scielo, MEDLINE / PubMed. The results were inserted in separate tables for better teaching and understanding of the research. It was concluded that physiotherapy has much broader resources than electrothermal phototherapy and kinesiotherapy, all the resources found can be applied inside and outside the environment of the physiotherapy clinic with specific objectives regarding the degree of OA.
\end{abstract}

Keywords: Elderly; Knee osteoarthritis; Physiotherapy.

\section{Resumen}

La osteoartritis de rodilla (OA) es una patología de orden degenerativo, que se produce debido a varios factores. En el mundo hay 50 millones de personas en esta condición, la fisioterapia tiene varias técnicas para tratar a estos individuos entre las ya conocidas, la electrofototermoterapia y la kinesioterapia. El estudio busca enfoques que puedan aplicarse en la práctica clínica basada en la evidencia. La investigación surge a través de la estrategia PICO: Ancianos, recursos terapéuticos, tratamiento conservador, osteoartitis de rodilla. Aplicación de un operador booleano AND para maximizar los resultados, con 268 artículos encontrados en Google Scholar, Scielo, MEDLINE / PubMed. Los resultados se insertaron en tablas separadas para una mejor enseñanza y comprensión de la investigación. Se concluyó que la fisioterapia tiene recursos mucho más amplios que la fototerapia electrotérmica y la kinesioterapia, todos los recursos encontrados se pueden aplicar dentro y fuera del entorno de la clínica de fisioterapia con objetivos específicos en cuanto al grado de OA.

Palabras clave: Ancianos; Artrosis de rodilla; Fisioterapia. 


\section{Introdução}

Segundo Cobra e Ruiz (1956, p.1) a osteoartrite (AO), "é uma doença degenerativa crônica das articulações: caracteriza-se por alteração estrutural e erosão das superfícies cartilaginosas articulares, proliferação óssea marginal e arborização das extremidades [...]" Os autores também afirmam que o tratamento cirúrgico é uma opção, mas não ressaltaram na época a fisioterapia, provável pela limitação da época.

O Consenso Brasileiro para Tratamento da Osteoartrite, afirma que essa afecção é de ordem evolutiva. Sendo multifatorial, o tratamento é amplo e com melhora significativa na maioria dos casos. O tratamento inicialmente pode ser nãofarmacológico; com o encaminhamento para fisioterapia e educação em saúde. A respeito da perda de peso deve-se manter a reeducação alimentar e hábitos saudáveis. Esse tratamento também pode ser associado ao uso de fármacos. Quando não há prognóstico favorável, é indicado o tratamento cirúrgico. (Coimbra et al., 2002).

Cobra e Ruiz (1956), aponta que os fatores que predispunham pacientes são: hereditariedade, comorbidades, idade, postura, infecções, queda de hormônios. Sabe-se hoje que as comorbidades e sexo estão alinhadas no que diz respeito a essa afecção, pois diversos estudos apontam que mulheres, obesidade e hipertensão estão atreladas frequentemente a esses casos.

No Brasil não existe dados precisos a respeito da incidência concreta, mas propõe que no mundo mais de 50 milhões de pessoas estão com o diagnóstico de OA e geralmente possuem idade maior que 50 anos. A adesão ao sistema de saúde pelo paciente idoso é complicada quando se considera alguns fatores; a mobilidade, fragilidade, condição financeira e social. (Coimbra et al., 2004.)

O IBGE (2008), aponta que no Brasil, há cerca de 19 milhões de indivíduos com mais de 60 anos, afirmam ainda que esse número irá elevar-se até 2050. O tratamento da OA é multimodal e o paciente deve ser educado em relação às mudanças dos hábitos de vida, concordando a ideia supracitada a respeito das características do tratamento. (Rezende, Campos \& Paulo, 2013).

Mulheres idosas, são as que buscam o sistema de saúde com mais frequência. Elas possuem necessidade de auxílio de companhia para locomoção muito embora isso não mude o quadro geral. Tal condição pode ser explicado devido às mudanças hormonais pelas quais as mulheres passam ao longo da vida, de modo que após a menopausa o corpo sofre mudanças radicais e o sistema hormonal atua em baixa, essas alterações necessitam ser acompanhadas por especialistas. (Cruz, Vieira, Carneiro, Costa \& Caldeiro, 2020).

Um a cada 12 indivíduos tem OA, sendo a sua maioria mulheres, quando passam da menopausa, por vezes a afecção surge ainda durante a vida ativa da paciente, essas condições foram evidenciadas no estudo, onde demonstra que tal condição surge durante os 60 a 70 anos, junto a isso podem surgir os processos de osteopenia, osteoporose, artrite reumatoide. Concluise que a condição pode surgir em decorrência da qualidade física do indivíduo. (Pancotte et. al., 2017).

Quando submetidas a avaliação de funcionalidade para adesão a fisioterapia, mulheres idosas apresentam com frequência dor e diminuição da funcionalidade. Por vezes junto a esses sinais e sintomas, apresentam rigidez articular, fraqueza, cinesiofobia, crepitação, quadros esporádicos de perca repentina da força. (Mascarenhas, Campos, Azevedo \& Reis, 2010).

O estudo realizado por Santos, Damianni, Marcon, Haupenthal e Avelar (2020), avaliou a funcionalidade e qualidade de vida de mulheres idosas com OA, e determinaram que a dor é o sintoma de maior frequência nas mulheres, seguido de rigidez articular e inflamação. Estes sintomas limitantes, afetam a velocidade da marcha, causa perda de força e equilibro, podendo apresentar-se poliqueixosa.

Através de revisão sistemática realizado por Duarte et al. (2013), foi constatado que o exercício físico é uma conduta eficaz no tratamento da OA, visto que todos os artigos inseridos naquele estudo apontavam resultados significantes na melhora 
da dor, função, mobilidade, flexibilidade, força. Portanto, é fato que a atividade física deve ser aliada às condutas terapêuticas e essa é apenas uma conduta no arsenal que pode ser usado no tratamento.

A fisioterapia possui diversas técnicas para lidar com esse caso, seja de forma ativa ou passiva. O fisioterapeuta deve ter o olhar clínico amplo no que se refere as condutas utilizadas com esses pacientes. É fato que a fisioterapia atua de forma ativa e consistente no tratamento dessa patologia que não tem cura, mas pode prolongar a saúde articular e global. Os recursos são diversos e será observável as técnicas presentes nas bases de dados que podem ser utilizadas no tratamento dessa patologia, tratamentos estes que podem ir além de cinesioterapia e eletrotermofototerapia. (Cunha, Silva \& Silva, 2017).

Diante das afirmações acima, esta pesquisa é realizada com foco em responder a seguinte questão: Quais são os resultados apresentados nos estudos sobre relacionados a análise dos recursos terapêuticos em pacientes idosos com osteoartrite do joelho? Desta forma, é realizada uma pesquisa quali-quantitativa, de caráter exploratório, de modo a investigar os artigos relacionados e apresentar os seus resultados.

Com isso, o objetivo geral é analisar os resultados dos estudos para obter conclusões dos melhores métodos terapêuticos para pacientes idosos com osteoartrite no joelho. Para alcançar o objetivo geral, os objetivos específicos são: apresentar a análise quantitativa dos artigos relacionados e apresentar a discussão dos resultados desses estudos. Espera-se que através desta pesquisa, seja possível responder à questão desta pesquisa, de modo a enriquecer a literatura acadêmica e o conhecimento do leitor.

\section{Metodologia}

Trata-se de uma pesquisa quali-quantitativa de caráter explicativo. Para o desdobramento desta pesquisa, utilizou-se a estratégia PICO: idosos, recursos terapêuticos, tratamento conservador, osteoartrite do joelho. Foi aplicando os termos "idoso", "osteoartrite do joelho" e "fisioterapia", respectivamente, nas bases Google Scholar, Scientific Eletronic Library Online (Scielo) e MedLine/PubMed, utilizando-se do operador booleano AND.

Foram encontrados nas respectivas ordens, 108 resultados, 3 resultados e 157 resultados. Foram excluídos artigos de caráter pago ou acesso restrito, cartilhas, folhetos e publicações de blogs. Foram contabilizados somente aqueles que estavam disponíveis de forma integral e que tinham relação direta com a população a ser pesquisada.

Os artigos incluídos foram compreendidos e resumidos para serem tabulados e divididos no Quadro 1, que corresponde aos achados na base Google Scholar; Quadro 2, artigos da base Scielo e Quadro 3, com artigos da MedLine/PubMed. Tal distribuição confere a melhor compreensão das informações obtidas em cada base de dados, o que consequentemente irá melhorar a didática da pesquisa. 
Figura 1- Fluxograma PRISMA de seleção dos estudos que constituíram a amostra.

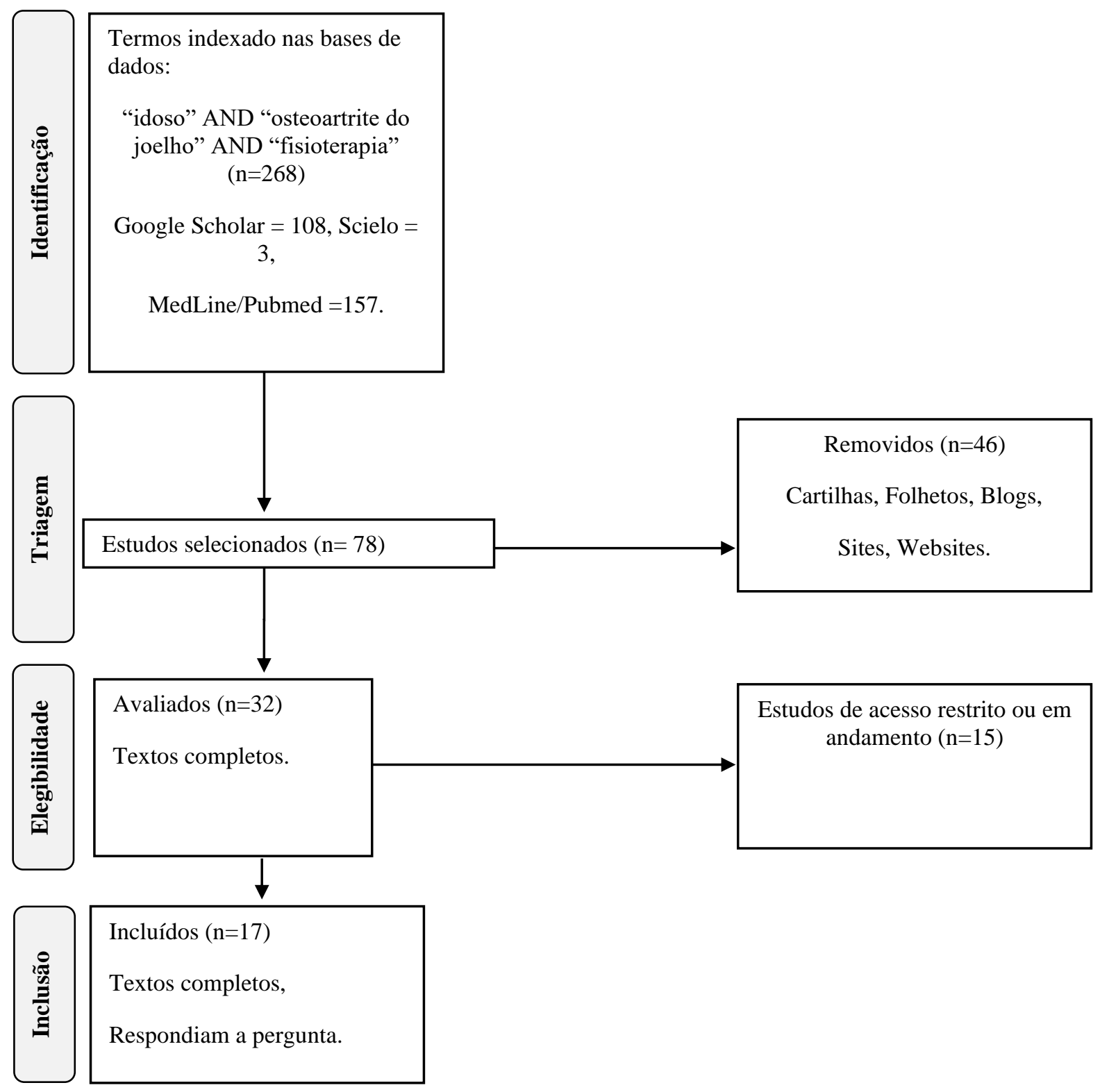

Fonte: Autores (2021).

\section{Resultados}

Nesta seção, será apresentado organizado em quadros, os artigos científicos encontrados.

No quadro 1 observa-se os artigos científicos encontrados na base Google Scholar, de acordo com título, autores e um breve comentário sobre o desfecho da pesquisa realizada. 
Quadro 1- Artigos científicos encontrados na base Google Scholar.

\begin{tabular}{|c|c|c|}
\hline Título & Autores & Comentário \\
\hline $\begin{array}{l}\text { A fisioterapia na saúde do idoso com } \\
\text { osteoartrite: uma revisão de literatura. }\end{array}$ & Cunha et al. (2017). & $\begin{array}{l}\text { Apontam outras técnicas além das já reconhecidas; } \\
\text { técnicas de acupuntura e vídeo-games são eficazes no } \\
\text { tratamento geral da OA. }\end{array}$ \\
\hline $\begin{array}{l}\text { Cinesioterapia como tratamento para } \\
\text { osteoatrite no joelho. }\end{array}$ & Sanchez et al. (2007). & $\begin{array}{l}\text { Cinesioterapia tem resultado positivo no tratamento da } \\
\text { OA e o fisioterapeuta deve ir além, observando } \\
\text { possíveis patologias secundárias. }\end{array}$ \\
\hline $\begin{array}{l}\text { Eficácia do treino de marcha e equilíbrio em } \\
\text { pacientes com osteoartrite de joelho. }\end{array}$ & Yamada et al. (2016). & $\begin{array}{l}\text { Exercícios de equilíbrio e treino de marcha contribuem } \\
\text { positivamente para o tratamento da OA resultando em } \\
\text { melhora da força, amplitude de movimento, qualidade } \\
\text { de vida. }\end{array}$ \\
\hline $\begin{array}{lllll}\text { Hidroterapia em idosos: Uma revisão } \\
\text { integrativa. }\end{array}$ & Silva et al. (2019). & $\begin{array}{l}\text { A hidroterapia é uma técnica positiva, com diversos } \\
\text { benefícios, diminuindo o risco de queda e contribuindo } \\
\text { para o envelhecimento saudável. }\end{array}$ \\
\hline $\begin{array}{l}\text { Utilização do kinesiotaping no recrutamento } \\
\text { muscular e redução da dor em pacientes com } \\
\text { osteoartrose grau } 1 \text { e } 2 \text { de joelho. }\end{array}$ & Bandeira et al. (2018). & $\begin{array}{l}\text { O kinesiotaping tem resultado positivo em relação a dor } \\
\text { e funcionalidade. Mas não para auxílio de excitação } \\
\text { motora de imediato. }\end{array}$ \\
\hline $\begin{array}{l}\text { Visão ortopédica do tratamento da gonartrose } \\
\text { nas fases iniciais. (Breve Revisão). }\end{array}$ & Ávila, et al. (2011). & $\begin{array}{l}\text { Terapia física na fase } 2 \text { com uso de termo terapia com } \\
\text { Ultrassom, laser, crioterapia, TENS, acupuntura, } \\
\text { musculação, pilates e hidroterapia. }\end{array}$ \\
\hline $\begin{array}{l}\text { Comparação do uso do frio e calor superficial } \\
\text { associados à cinesioterapia em indivíduos } \\
\text { com AO de joelho: um estudo piloto. }\end{array}$ & Neves et al. (2016). & $\begin{array}{l}\text { O uso de frio ou calor favorece o indivíduo em relação } \\
\text { a funcionalidade, mobilidade e força. Não sendo } \\
\text { possível confirmar a redução da dor com a } \\
\text { termoterapia. }\end{array}$ \\
\hline
\end{tabular}

Fonte: Autores (2021).

No Quadro 2 observa-se o único artigo encontrado na base Scielo, esse resultado foi mostrado em decorrência da aplicação do operador booleano AND. A mudança em uma única palavra o operador trará resultado diferente.

Quadro 2 - Artigo encontrado na base Scielo.

\begin{tabular}{|c|c|c|}
\hline Título & Autores & Comentário \\
\hline $\begin{array}{c}\text { Application of kinesiotherapy and } \\
\text { electrothermotherapy in the treatment of } \\
\text { elderly with knee osteoarthrosis: a } \\
\text { comparative study. }\end{array}$ & Almeida et al. (2016). & $\begin{array}{c}\text { Cinesioterapia aumenta a amplitude de } \\
\text { movimento do joelho e reduz a dor. } \\
\end{array}$ \\
& & $\begin{array}{c}\text { Eletroterapia com TENS e US terapêutico } \\
\text { reduz o nível da dor, por consequência } \\
\end{array}$ \\
\end{tabular}

Fonte: Autores (2021).

No Quadro 3 observam-se os artigos científicos encontrados na base MEDLINE/PubMed. Nele foram encontrados artigos de alta confiança, havia artigos relevantes para essa pesquisa, mas ainda em fase de aplicação, portanto foram 
excluídos.

Quadro 3 - Artigos científicos encontrados na base MEDLINE/PubMed.

\begin{tabular}{|c|c|c|}
\hline Título & Autores & Comentário \\
\hline $\begin{array}{l}\text { Treatment using } 448 \mathrm{kHz} \text { capacitive resistive } \\
\text { monopolar radiofrequency improves pain and } \\
\text { function in patients with osteoarthritis of the } \\
\text { knee joint: a randomised controlled trial. }\end{array}$ & Kumara e Watson (2019). & $\begin{array}{l}\text { A terapia por radiofrequência mostra resultados } \\
\text { positivos no que se refere a dor e qualidade de vida. } \\
\text { Mas sem resultado expressivo no que se refere ao } \\
\text { tempo de marcha. }\end{array}$ \\
\hline $\begin{array}{l}\text { Immediate Effects of Transcutaneous Electrical } \\
\text { Nerve Stimulation on Pain and Physical } \\
\text { Performance in Individuals With } \\
\text { Preradiographic Knee Osteoarthritis: A } \\
\text { Randomized Controlled Trial. }\end{array}$ & Shimoura et al. (2019). & $\begin{array}{l}\text { Aplicar TENS antes da radiografia mostra resultado } \\
\text { positivo na escala de dor e caminhada prolongada. }\end{array}$ \\
\hline $\begin{array}{l}\text { Immediate and short-term effects of gait } \\
\text { retraining on the knee joint moments and } \\
\text { symptoms in patients with early tibiofemoral } \\
\text { joint osteoarthritis: a randomized controlled } \\
\text { trial. }\end{array}$ & Cheung et al. (2018). & $\begin{array}{l}\text { Reeducar a marcha pode ser uma estratégia positiva } \\
\text { para o tratamento da OA, aliviam a dor articular e } \\
\text { melhorando a mobilidade desses indivíduos. }\end{array}$ \\
\hline $\begin{array}{l}\text { "I was really sceptical...But it worked really } \\
\text { well": a qualitative study of patient perceptions } \\
\text { of telephone-delivered exercise therapy by } \\
\text { physiotherapists for people with knee } \\
\text { osteoarthritis. }\end{array}$ & Lawford et al. (2018). & $\begin{array}{l}\text { Terapia por chamada telefônica prestando auxílio } \\
\text { em cinesioterapia mostra-se efetivo na melhora da } \\
\text { função e dor. }\end{array}$ \\
\hline $\begin{array}{l}\text { Aquatic Exercise for the Treatment of Hip and } \\
\text { Knee Osteoarthritis. }\end{array}$ & Franco et al. (2017). & $\begin{array}{l}\text { Hidroterapia no curto prazo auxilia na melhora da } \\
\text { QV e função, no longo prazo melhora a força, } \\
\text { função, flexibilidade, mobilidade e potencializa os } \\
\text { efeitos já citados no curto prazo. }\end{array}$ \\
\hline $\begin{array}{l}\text { Exercise, manual therapy, and use of booster } \\
\text { sessions in physical therapy for knee } \\
\text { osteoarthritis: a multi-center, factorial } \\
\text { randomized clinical trial. }\end{array}$ & Fitzgerald et al. (2016). & $\begin{array}{l}\text { Terapia manual e exercícios aplicados de forma } \\
\text { isolada não apresenta resultado significante no } \\
\text { curto prazo. }\end{array}$ \\
\hline $\begin{array}{l}\text { Joint Mobilization enhances mechanisms of } \\
\text { conditioned pain modulation in individual with } \\
\text { osteoarthritis of the knee. }\end{array}$ & Courtney et al. (2016). & $\begin{array}{l}\text { Mobilização articular com técnicas oscilatórias e } \\
\text { vibrações tem resultado positivo na melhora da dor. }\end{array}$ \\
\hline $\begin{array}{l}\text { The effects of exercise and neuromuscular } \\
\text { electrical stimulation in subjects with knee } \\
\text { osteoarthritis: a 3-month follow-up study. }\end{array}$ & Laufer et al. (2014). & $\begin{array}{l}\text { No longo prazo, aplicar EENM ou exercício isolado } \\
\text { traz efeitos semelhantes. Embora aplicar EENM no } \\
\text { curto prazo resulte em melhora da dor. }\end{array}$ \\
\hline
\end{tabular}

Fonte: Autores (2021).

Através da cinesioterapia, a eletrotermofototerapia já é reconhecida no tratamento da OA, evidenciada em diversos estudos. Cunha et al. (2017), em uma revisão de literatura, apontam a crâniopuntura, geoterapia e a postulada wiiterapia como técnicas eficazes. O crâniopuntura através de pontos específicos: alivia a dor, melhora a mobilidade e funcionalidade. Através de materiais terrosos, a geoterapia mostra-se eficaz no controle da OA, assim como a wiiterapia, que se assemelha a cinesioterapia usando videogames, são eficazes para o quadro geral da OA.

Apesar da limitação da época Sanchez, Ros, Silva e Uccio (2007), apontam a baixa evidência nas bases de dados, mas concluem que a cinesioterapia tem efeito positivo no ganho de amplitude de movimento, flexibilidade e atividade de vida diária. Seus resultados também orientam que o fisioterapeuta não deve focar só na causa da dor, mas também na inter-relação entre outros sistemas, articulações e/ou patologias que podem derivar de uma OA.

Um estudo realizado por Yamada, Munoz e Moura (2016), avaliaram o desempenho de 20 indivíduos com diagnóstico de OA dividindo-os em dois grupos. O primeiro foi proposto treino de marcha, através da caminhada em direções variadas com determinadas repetições. Já no segundo grupo foi realizado exercícios de equilíbrio unipodal, sobre superfície plana e irregular. Nos dois grupos eram inicialmente realizados alongamentos estáticos e dinâmicos no início das sessões. Os 
resultados foram positivos na melhora da dor, força, amplitude de movimento e qualidade de vida.

Silva, Brito, Santos, Cavalero e Holanda (2019), afirmam que a hidroterapia é uma técnica eficaz no tratamento da AO. Entretanto, é válido expor que os autores citados nesta pesquisa não possuem total concordância no que se refere aos benefícios para cada gênero, embora seja possível ver melhora do equilíbrio nos indivíduos estudados, mesmo que não seja evidenciado nada específico em relação às suas funcionalidades, contudo, a hidroterapia é uma técnica válida e faz parte das diversas técnicas positivas no tratamento da OA.

Bandeira, Roque e Comel (2018), avaliaram o desempenho de 13 mulheres idosas com AO, através do Kinesio Taping (técnica de reabilitação desenvolvida para facilitar o processo de regeneração do organismo) durante o agachamento. Os pontos avaliados foram: dor, questionário Licholm e eletromiografia. Os resultados mostraram-se positivos nos dois primeiros pontos, contudo, o resultado da eletromiografia não demonstrou resultados significantes. Apesar dos achados, os autores concordam positivamente com melhoras em relação à dor, no exercício de subir e descer escadas, travamento e instabilidade. Também foi apontado resultados insignificantes para eletromiografia do músculo vasto medial e vasto lateral.

Ávila e Sá (2011), explicam que a OA do joelho é faseada, sendo que na primeira, deve ser realizada reeducação de hábitos, afirmando a perda de peso como fator primordial para manter a saúde articular. Os autores também afirmam que na fase 2, a terapia física deve ser iniciada através de recursos eletrofototermoterapêuticos, acupuntura, musculação, pilates e hidroterapia. Já na fase 3, é preferível o tratamento medicamentoso intra-articular. Neste estudo é importante salientar que os recursos terapêuticos não são citados.

Buscando avaliar a efetividade da termoterapia com uso de frio ou calor em indivíduos com AO de joelho, Neves, Kruger, Oliveira e Alfieri (2016), avaliaram 9 participantes, dividindo 3 participantes no grupo de cinesioterapia associada ao frio e 6 participantes no grupo de cinesioterapia associada ao calor. A análise teve foco em testes específicos de funcionalidade, dor, amplitude de movimento e dinamometria. Conclui-se que os recursos termoterápicos são eficazes na funcionalidade, mobilidade e força. Entretanto, não foi constatado a redução da dor.

Almeida, et al. (2016), realizou um estudo com dois grupos de idosas com diagnóstico de OA do joelho. Para o grupo 1, descrevem o alongamento passivo de isquiostibiais, tensor da fáscia lata, tríceps sural, além de outras técnicas ativo e passivo. O grupo 2, fazia o uso de eletroterapia com TENS e US terapêutico, especificando o uso de TENS no modo acupuntura por 30 minutos e o US sem cálculo específico. Os resultados não foram significativos para determinar a eficácia real de cada conduta tomada nesse estudo.

Kumara e Watson (2019), avaliaram 45 indivíduos, dividindo-os em três grupos: controle, falso e placebo. Foi aplicando no grupo controle: radiofrequência em $448 \mathrm{kHz}$ e atendimento padrão; no falso, somente atendimento padrão e no grupo placebo, não recebia estímulo de energia nem foi solicitado feedback verbal. Os resultados mostraram-se positivos no grupo controle e placebo, mostrando melhora no quadro da dor e qualidade de vida; já o teste de levantar e andar mostrou-se inalterado nos três grupos.

Shimoura, Lijima, Susuki e Aoyama (2019), realizaram aplicação de TENS antes de uma radiografia, através de testes de levantar e andar, caminhada de 6 minutos, EVA e questionário JKOM. Foi avaliado 50 indivíduos, distribuindo-os em dois grupos de 25. Em um grupo era aplicado o TENS e no outro grupo era aplicado TENS sem corrente elétrica. Os resultados mostraram-se positivos, apenas em relação à caminhada de 6 minutos e EVA. Os demais pontos avaliados na pesquisa mostraram-se com alteração irrelevante para ter significância.

De acordo com Cheung et al. (2018), a reeducação da marcha com biofeedback produz efeito positivo na melhora da dor e prolonga a função dos indivíduos, se comparando com a marcha livre. Através desta pesquisa, foi possível observar que a reeducação da marcha reduz a pressão intra-articular medial do joelho, produzindo efeitos benéficos.

Lawford, Dellane, Benell e Hinman (2018), avaliaram o desempenho de fisioterapeutas prestando assistência 
telefônica com exercício ativo, onde o paciente mesmo a distância realizava todo seu atendimento de forma independente. Cada integrante da pesquisa recebeu material físico: fita e elástico para o tratamento, além do material digital: sites, vídeos e chamadas de vídeos para acompanhamento. O desfecho mostrou-se positivo, pois os indivíduos relataram melhora na força, confiança para realizar suas atividades, e redução da dor.

Através de uma revisão sistemática de altíssima relevância, Franco, Morelhão e Carvalho (2017), apontam a hidroterapia como tratamento eficaz para indivíduos com OA no curto prazo, contribuindo na melhora da qualidade de vida e função. No longo prazo, pode-se afirmar que a hidroterapia contribui para aumento de força, confiança e mobilidade, além dos pontos supracitados potencializados no estudo.

Fitzgerald et al. (2016), avaliaram trezentos indivíduos, distribuindo-os em quatro grupos; exercícios sem esforço, com esforço, terapia manual + exercício sem esforço, terapia manual + exercícios com esforço. Os achados não foram estatisticamente promissores, pois, tanto no curto prazo, a $\mathrm{TM}+$ exercícios de força, como na $\mathrm{TM}+$ exercício de esforço, os achados mostram-se insignificantes.

Courtney, Steffen, Penas, Kim e Chmell (2016), propôs a técnica de mobilização articular em 38 indivíduos com mobilização oscilatória e descompressão para redução da dor. Esta técnica, contribuiu positivamente no que se refere a percepção da dor. Também foi avaliado a percepção da vibração oscilatória durante as técnicas de mobilização, porém não foi notado diferença significante.

Aplicar a eletroestimulação elétrica neuromuscular (EENM) ou apenas exercícios de fortalecimento isolado traz efeitos igualmente significantes no longo prazo. Foi o que evidenciaram Laufer, Shtraker e Gabyzon, (2014). Os autores também afirmam que a EENM no curto prazo, traz efeito positivo no tratamento da dor. É importante explicar que, a aplicação de corrente elétrica possui modos específicos para determinadas condições.

\section{Discussão}

Saindo da fisioterapia tradicional, os estudos propostos por Cunha et al. (2017) e Lawford et al. (2018), incluem de forma positiva o tratamento por meio eletrônico. Tanto no exercício ativo frente ao vídeo game, como por telefonemas, ambos possuem efeitos satisfatórios. Vale ressaltar que a metodologia aplicada nesses estudos foi planejada e com a distribuição de recursos específicos para casos específicos. A evolução do mercado eletrônico pode contribuir positivamente no futuro. Hoje, os recursos são limitados e não podem ser aplicados a todos os casos de $\mathrm{AO}$ e por ser uma doença de caráter evolutiva e degenerativa, pode pôr em risco a saúde daquele paciente, portanto, a avaliação e aplicação de qualquer técnica através de dispositivos eletrônicos deve ser objetiva e individualizada.

Um estudo mais antigo realizado por Silva e Sá (2011), é apontado os recursos eletrotermofototerapêuticos dispostos para tratamento dessa afecção, que possuem efeito positivo quando aplicados em osteoartrite grau 2. Já Almeida et al. (2016), explica que os mesmos recursos trazem resultados significativos na melhora da dor e amplitude de movimento do joelho. Shimoura et al. (2019), contribui para esses argumentos citando como benefício destes recursos, a melhora da caminhada.

Yamada et al. (2016), afirma que o treino de marcha e equilíbrio traz efeitos positivos na dor, força, qualidade de vida. Cheung et al. (2018), também afirma que o treino de marcha tem bom resultado no quadro de dor, além de incluir a mobilidade articular. Os autores comunicam-se indiretamente entre si ao afirmar que o treino de marcha nos indivíduos com OA tem resultado benéfico. Vale ressaltar que a marcha é uma técnica da cinesioterapia clínica, com foco específico no alívio da pressão do compartimento do joelho.

Franco et al. (2017) e Silva et al. (2019), expõem conclusões similares a respeito da hidroterapia em idosos com AO. Eles afirmam que no longo prazo, a técnica auxilia positivamente na melhora da dor, força e por consequência melhora a saúde 
articular, contribuindo para outros benefícios como a diminuição do risco de queda, envelhecimento saudável e longevidade da saúde articular.

Partindo para o âmbito da terapia manual os autores Fitzgerald et al. (2016) e Courtney et al. (2016), compararam o efeito com sentidos postos. O primeiro, avaliou a TM associando a exercícios de forma isolada e não apresentou resultado positivo. O segundo apresentou resultado satisfatório propondo técnica de TM articular como eficaz na melhora da dor.

Kumara e Watson (2019), definem que a terapia por radiofrequência por onda específica de $448 \mathrm{kHz}$ não apresenta resultado expressivo, mas contribui para melhora da dor. Laufer et al. (2014), avaliaram a eletroestimulação neuromuscular no curto e longo prazo e definiu que no curto prazo, essa contribui para o alívio da dor, embora no longo prazo não haja resultado expressivo. Ambos os autores utilizaram meios não tão reconhecidos na prática clínica para tratamento da OA, mas foi possível notar que dentro de cada técnica há efeitos positivos.

Abreu et al. (2020), avaliou a aplicação do laser terapêutico em pacientes com OA, e os achados foram promissores no que diz respeito aos questionários (Womac e Lesquene) aplicados sobre funcionalidade. Os artigos descritos nessa pesquisa podem ser aplicados em conjunto para uma evolução benéfica ao paciente se tratando do quadro geral, visto que dor, rigidez e diminuição da funcionalidade, são características em sua maioria presente nesses casos.

Bandeira et al. (2018), aplicaram a Kinesio Taping para auxiliar no recrutamento muscular. Considerando esta finalidade, não houve resultado positivo, mas em relação à dor e mobilidade, foi notado que os indivíduos melhoraram as escalas avaliadas na pesquisa. Neves et al. (2016), ao utilizar o frio ou calor (termoterapia), não traz resultados positivos com relação à dor, entretanto, aplicar qualquer um dos dois possui resposta significativa na mobilidade.

Nos estudos de intervenção, foram observados o uso de questionários específicos para avaliar dor e função, pensando nisso Metsavaht, Liporase, Sposito e Riberto (2011), sugeriu os questionários WOMAC para avaliar a funcionalidade. Com isso, constatou-se que o IKDC pode ser utilizado para avaliar limitações funcionais. Conforme a metodologia proposta, é possível compreender que se utilizados juntos para avaliar tal paciente, os resultados serão mais sólidos e trarão mais detalhes a respeito da condição do paciente.

Bakirhan, Bozan, Unver e Karatosun (2017), concluiu que fatores como obesidade e falta de atividade física estão atreladas às condições que predispõe à AO. Também foi possível constatar que não há relação entre idade e grau de OA, o que não se repete ao comparar o índice de massa corporal (IMC) e dor, ou seja, pacientes com IMC alto apresentavam quadro de dor mais elevado em relação àqueles que estavam com um IMC menor.

Os pacientes que evoluem para o processo cirúrgico, apresentam comorbidade decorrente da obesidade e hipertensão, foi o que registrou Bezerra, et al. (2017). Através do perfil clínico de pacientes submetidos a artroplastia total de joelho, ficou evidente que as mulheres são as mais afetadas, não havendo relação entre fumantes e não fumantes, onde todos apresentam os sinais típicos de um sujeito com OA.

Fernandes, Poeta, Martins, Lima e Neto (2018), avaliou a qualidade de vida em pacientes que não tiveram sucesso com o tratamento conservador, em relação ao equilíbrio e força. Nos resultados, não foi possível notar diferença expressiva se comparando a grupo de pessoas saudáveis, entretanto, ressalta-se que o próprio sujeito apresenta melhora nesses aspectos. Se comparado com o pré cirúrgico, o resultado mais significante no longo prazo é a melhora da dor e funcionalidade.

\section{Conclusão}

Conforme os estudos relacionados, foi possível concluir que a fisioterapia possui uma gama diversificada de tratamentos que tem resultados positivos na OA de joelho. Os recursos que podem ser aplicados vão além dos reconhecidos pelos pacientes como "choquinho" e "exercício" na clínica de fisioterapia, para tratar de modo mais eficaz, evitando desta 
forma o abandono do paciente ao tratamento. A literatura tem permitido ampliar os horizontes sobre todos os aspectos dessa doença que afeta os joelhos da população idosa. Diversas técnicas são publicadas anualmente para o tratamento, é importante ao profissional de fisioterapia manter-se atualizado e basear suas condutas em evidências concretas, buscando garantir a saúde articular do paciente.

Este estudo foi realizado através de uma análise dedicada das bases de dados com os indexadores e operadores booleanos, para minimizar os resultados e mesmo utilizando tal metodologia para a pesquisa ainda foram apresentados diversos artigos que fugiam a temática proposta, resultando num tempo maior de pesquisa e fator de resposta e exclusão expressivo.

Dessa forma, sugere-se que novos estudos sejam realizados buscando termos contemporâneos e outros operadores booleanos para encontrar resultados diversificados das pesquisas. Precisamos de pesquisas sobre melhores exercicios para o controle dos sintomas de OA de joelho em pacientes de todas as idades.

\section{Referências}

Abreu, T. S., Sanchez, E. M. G., Kuriki, G. M, Oshiro, J. M., Boldrini, R. C. B. C. \& Morimoto, M. M. (2020). Os benefícios da laserterapia de baixa intensidade associados a exercícios domiciliares em idosos com osteoartrite de joelho. Revista Pesquisa em Fisioterapia, 10 (1), 9p. https://doi.org/10.17267/2238-2704rpf.v10i1.2617

Almeida, F. J. F., Araújo, A. E. R., Carvalho, C. A., Fonseca, P. C. A., Nina, V. J. \& Mochel, E. (2016). Application of kinesiotherapy and electrothermotherapy in the treatment of elderly with knee osteoarthrosis: a comparative study. Fisioterapia em movimento, 29 (2), 325-334. Curitiba. https://www.scielo.br/j/fm/a/MMcjSqwb7g7LqqXpQnNYHXs/?format=pdf\&lang=en

Ávila, Sérgio S. S., \& Marcus V. C. M. C. (2011). Visão ortopédica do tratamento da gonartrose nas fases iniciais. RBM revista brasileira de medicina,68 (4).

Bakirhan, S., Bozan, O., Unver, B. \& Karatosun, V. (2017). Evaluation of functional characteristics in patients with knee osteoarthritis. Acta ortopédica brasileira, 25 (6), 248-252. https://doi.org/10.1590/1413-785220172506144577

Bandeira, E., Roque, J. A. \& Comel, J. C. (2018). Utilização do kinesio taping no recrutamento muscular e redução da dor em pacientes com artrose grau 1 e 2 de joelho. Revista Saúde Integrada, 11, (21), 82-94. https://core.ac.uk/download/pdf/229765921.pdf

Bezerra, M. J. M. C., Barbosa, I. M., Sousa, T. G., Fernandes, L. M., Maia, D. L. M. \& Holanda, L. M. (2017). Profile of patients receiving total knee arthroplasty: a abrecross-sectional study. Acta ortopopédica brasileira, 25 (5), 202-205. https://doi.org/10.1590/1413-785220172505168806

Cheung, R. T. H., Au, I. P. H., Zhang, J. H., Chan, Z. Y. S, Delúzio, K. \& Raiwbown, M. J. (2018). Immediate and short-term effects of gait retraining on the knee joint moments and symptoms in patients with early tibiofemoral joint osteoarthritis: a randomized controlled trial. Science Direct: Osteoarthritis and Cartilage, 26 (11), 1479-1486. https://www.sciencedirect.com/science/article/pii/S1063458418313864

Cobra, C. J. \& Ruiz, S. I. D. (1956). Osteoartrite. Revista de Medicina, 40 (1), 104-110.

Coimbra, I. B., Pastor, E. H., Greve, M. D., Puccinelli, M. L. C., Fuller, R., Cavalcanti, F. S., Maciel, F. M. B. \& Honda, E. (2002). Consenso brasileiro para o tratamento da osteoartrite (artrose). Revista brasileira de reumatologia, 42 (6), 371-374. http://cidmed.com.br/pdf/osteoartrite.pdf

Coimbra, I. B., Pastor, E. H., Greve, M. D., Puccinelli, M. L. C., Fuller, R., Cavalcanti, F. S., Maciel, F. M. B. \& Honda, E. (2004). Osteoartrite (artrose): tratamento. Revista brasileira de reumatologia, 44, (6), 450-453. https://www.scielo.br/j/rbr/a/F39LTRWZ985dPVQTpYPcvfJ/?format=pdf\&lang=pt

Courtney, C., Steffen, A. D., Penas, C. F., Kim, J. \& Chmell, S. (2016). Joint Mobilization enhances mechanisms of conditioned pain modulation in individual with osteoarthritis of the knee. Journal of Orthopaedic \& Sports Physical Therapy, 46 (3), 168-176. https://www.jospt.org/doi/10.2519/jospt.2016.6259

Cruz, P. K. R., Vieira, M. A., Carneiro, J. A., Costa, F. M. \& Caldeira, A. P. (2020). Difficulties of access to health services among non-institutionalized older adults: prevalence and associated factors. Revista brasileira de geriatria e gerontologia, 23, (6). https://doi.org/10.1590/1981-22562020023.190113

Cunha, A., Silva, C. N. \& Silva, M. K. (2017). A fisioterapia na saúde do idoso com osteoartrite: uma revisão de literatura. Revista Interdisciplinar de Ensino, Pesquisa e Extensão-REVINT, 5 (1), 130-143.

Duarte, V. S., Santos, M. L., Rodrigues, K. A., Ramires, J. B., Âreas, G. P. T. \& Borges, G. F. (2013). Exercícios físicos e osteoartrose: uma revisão sistemática. Revista Fisioterapia em Movimento, 26 (1), 1-10. https://doi.org/10.1590/S0103-51502013000100022

Faloppa, F. \& Belloti, J. C. (2006). Tratamento clínico da osteoartrose: evidências atuais. Revista brasileira de ortopedia. 41(3), 47-53. https://rbo.org.br/detalhes/33/pt-BR/tratamento-clinico-da-osteoartrose--evidencias-atuais

Franco, M. R., Morelhão, P. K. \& Carvalho, R. A. (2017). Aquatic exercise for the treatment of hip and knee osteoarthritis. Revista Physical therapy, 97, (7), 693-697.

Fernandes, D. A., Poeta, L. S., Martins, C. A. Q., Lima, F. \& Neto, F. R. (2018). Balance and quality of life after total knee arthroplasty. Revista brasileira de ortopedia, 53 (6), 747-753. https://doi.org/10.1016/j.rboe.2017.07.013 
Fitzgerald, G. K., Fritz, J. M., Chils, J. D., Brennan, G. P., Talisa, V., Neilson, B. D. \& Abott, J. H. (2016). Exercise, manual therapy, and use of booster sessions in physical therapy for knee osteoarthritis: a multi-center, factorial randomized clinical trial. Science Direct - Osteoarthritis and Cartilage, 24 (8), 1340-1349. https://www.sciencedirect.com/science/article/pii/S106345841601058X

Imoto, A. M., Peccin, M. S., \& Trevisani, V. F. M. (2012). Exercícios de fortalecimento de quadríceps são efetivos na melhora da dor, função e qualidade de vida de pacientes com osteoartrite do joelho. Acta ortopédica brasileira, 20(3), 174-179. https://doi.org/10.1590/S1413-78522012000300008

Kumaran, B. \& Watson, T. (2019). Treatment using $448 \mathrm{kHz}$ capacitive resistive monopolar radiofrequency improves pain and function in patients with osteoarthritis of the knee joint: a randomised controlled trial. National library of medicine, Physiotherapy, 105 (1), 98-107. https://www.intact.si/uploads/docs/Osteoarth-Indiba-Kumaran-Watson.pdf

Koche, J. C. (2011). Fundamentos da metodologia científica. Petrópolis. Vozes.

Laufer, Y. Shtraker, H. \& Gabyzon M. E. (2014). The effects of exercise and neuromuscular electrical stimulation in subjects with knee osteoarthritis: a 3month follow-up study. Clinical interventions in aging, 2 (1), 1153-1161. https://www.dovepress.com/the-effects-of-exercise-and-neuromuscular-electricalstimulation-in-su-peer-reviewed-fulltext-article-CIA

Lawford, B. J., Dellane, C., Benell, K. L. \& Hinman, R. S. (2018). "I was really sceptical...But it worked really well”: a qualitative study of patient perceptions of telephone-delivered exercise therapy by physiotherapists for people with knee osteoarthritis. Science Direct - Osteoarthritis and Cartilage, 26 (6), 741-750. https://doi.org/10.1016/j.joca.2018.02.909

Mascarenhas, C. H. M. Campos, S. L., Azevedo, L. M., \& Reis, N. M. J. (2010). Avaliação funcional de idosas com osteoartrite de joelho submetidas a tratamento fisioterapêutico. Revista baiana de saúde pública, 34 (2), 56-254. https://doi.org/10.22278/2318-2660.2010.v34.n2.a32

Mattos, F., Wolf, R., Peniche, M. L. F., Costa, S. N., Sabchuc, R. A. C. \& Bento, P. C. B. (2015). Comparação da funcionalidade, agilidade e equilíbrio dinâmico de idosas com e sem osteoartrite de joelhos. Revista educação física de Maringá, 26 (3), 435-441. https://doi.org/10.4025/reveducfis.v26i3.25254

Metsavaht, L., Leporace, G., Sposito, M. A. M. \& Riberto, M. (2011). Qual o melhor questionário para avaliar os aspectos físicos de pacientes com osteoartrite no joelho na população brasileira? Revista brasileira de ortopedia, 46 (3), 256-261. https://doi.org/10.1590/S0102-36162011000300004

Neves, H. C. C., Kruger, M. D., Oliveira, N. C. \& Alfieri, F. M. (2016). Comparação do uso do frio e calor superficial associados à cinesioterapia em indivíduos com osteoartrite de joelho: um estudo piloto. Revista digital, 20,(214). https://www.efdeportes.com/efd214/frio-e-calor-com-osteoartrite-dejoelho.htm

Oliveira, J. G. D., Frota, D. G., Barbieri, D. G. F. V., Sousa, M. E., Castro, S. \& Coutinho, S. M. A. (2015). Uso da eletroterapia associado a cinesioterapia no tratamento de pacientes com osteoartrite. O efeito da eletrolipofores no fibroedemagelóide. Revista Fisioterapia Ser, 10, (2), 5-103.

Pancotte, J. Bortoluzzi, E. C., Graeff, D. B., Sant'Anna A. A. L., Wibelinger, L. M., \& Doring, M. (2017). Osteoartrite: prevalência e presença de fatores de risco em idosos ativos. Revista de Ciências Médicas e Biológicas, 16 (1), 40-44. https://doi.org/10.9771/cmbio.v16i1.17418

Rezende, M. U., Campos, G. C. \& Paulo, A. F. (2013). Conceitos atuais em osteoartrite. Acta ortopédica brasileira, 21 (2), 120-122. São Paulo: Fmusp. https://doi.org/10.1590/S1413-78522013000200010

Rodrigues, R. C., Pontin, J. C. B., Falcon, S. M. \& Chamlian, T. R. (2013). Avaliação do perfil, satisfação e efetividade do tratamento fisioterapêutico em grupo nos pacientes com osteoartrite de joelho. Acta Fisiátrica, 20 (3), 124-128. https://doi.org/10.5935/0104-7795.20130020

Sanchez, F. F., Ros, R. C. M. M., Silva, T. R. \& Uccio, C. B. (2007). Cinesioterapia como tratamento para osteoartrite no joelho. In: Omnia Humanas, 4 (2), 174. Revista Omnia Saúde. Adamantina, SP: Unifai. http://www.fai.com.br/portal/_arquivos/_itens_home/ce98956894fb81441f524213900eda13.pdf\#page=30

Santos, M. G., Damianni, P., Marcon, A. C. Z., Haupenthal, A. \& Avelar, N. P. C. (2020). Influence of knee osteoarthritis on functional performance, quality of life and pain in older women. Fisioterapia e movimento, 33. https://doi.org/10.1590/1980-5918.033.AO06

Shimoura, K., Lijima, H., Susuki, Y. \& Aoyama, T. (2019). Immediate effects of transcutaneous electrical nerve stimulation on pain and physical performance in individuals with preradiographic knee osteoarthritis: a randomized controlled trial. Archives of Physical Medicine and Rehabilitation, 100 (2), 300-306. https://pubmed.ncbi.nlm.nih.gov/30315763/

Silva, F. V., Brito, C. B., Santos, I. C. A., Cavalero, L. A. S. \& Holanda, T. M. (2019). Hidroterapia em idosos: uma revisão integrativa. In: Revista Biomotriz, 13 (4), 125-132. Cruz Alta, RS: Unicruz. https://www.revistaeletronica.unicruz.edu.br/index.php/biomotriz/article/download/78/30/238

Yamada, E. F., Muñoz, F. M. A \& Moura, P. M. (2016). Eficácia do treino de marcha e de equilíbrio em pacientes com osteoartrite de joelho. Fisioterapia brasileira, 17 (4), 313-320. https://portalatlanticaeditora.com.br/index.php/fisioterapiabrasil/article/view/504 